\title{
Depth of Response was Associated with Progression-Free Survival in Patients with Advanced Non-small Cell Lung Cancer treated with EGFR-TKI
}

\author{
Yu-Tao Liu', Kai Zhang ${ }^{2}$, Cheng-Cheng $\mathrm{Li}^{3}$, Xing-Sheng Hu${ }^{1}$, Jun Jiang ${ }^{4}$, Xue-Zhi Hao ${ }^{1}$, Yan Wang1, \\ Jun-Ling Li1 ${ }^{1}$ Pu-Yuan Xing1, Sheng Yang1, Xin Zhang1, Guo-Qiang Wang ${ }^{3}$, Shang-Li Cai ${ }^{3}$, Yuan-Kai Shi1 ${ }^{\bowtie}$ \\ 1. Department of Medical Oncology, Beijing Key Laboratory of Clinical Study on Anticancer Molecular Targeted Drugs, National Cancer Center/ National \\ Clinical Research Center for Cancer/ Cancer Hospital, Chinese Academy of Medical Sciences \& Peking Union Medical College, Beijing, P.R. China \\ 2. Cancer Center of Union Hospital, Tongji Medical College, Huazhong University of Science \& Technology, Wuhan, P.R. China \\ 3. The Medical Department, 3D Medicines Inc, Shanghai, P.R. China \\ 4. Department of radiology, Beijing Key Laboratory of Clinical Study on Anticancer Molecular Targeted Drugs, National Cancer Center/ National Clinical \\ Research Center for Cancer/ Cancer Hospital, Chinese Academy of Medical Sciences \& Peking Union Medical College, Beijing, P.R. China
}

$\square$ Corresponding author: Yuankai Shi, Department of Medical Oncology, Beijing Key Laboratory of Clinical Study on Anticancer Molecular Targeted Drugs, National Cancer Center/ National Clinical Research Center for Cancer/ Cancer Hospital, Chinese Academy of Medical Sciences \& Peking Union Medical College, Beijing, 100021, China. Tel: +86 108778 8293; Email: syuankai@cicams.ac.cn

(c) The author(s). This is an open access article distributed under the terms of the Creative Commons Attribution License (https://creativecommons.org/licenses/by/4.0/). See http:/ /ivyspring.com/terms for full terms and conditions.

Received: 2019.01.23; Accepted: 2019.07.28; Published: 2019.08.28

\begin{abstract}
Background: Response Evaluation Criteria in Solid Tumors (RECIST) has been widely utilized to evaluate new therapeutic strategies in cancer. However, RECIST fails to assess the heterogeneity of response in highly active therapies. Depth of response (DepOR), defined as the maximum percentage change in tumor size compared with baseline, may provide a new strategy to evaluate disease response. In the present study, we studied the association between DepOR and progression-free survival (PFS) in patients with advanced non-small cell lung cancer (NSCLC) treated with epidermal growth factor receptor tyrosine kinase inhibitor (EGFR-TKI).

Methods: Advanced NSCLC patients harboring EGFR driver mutation (L858R or exon 19 deletion) treated with EGFR-TKI from August 2014 to July 2017 from two sites were retrospetively collected for analysis. Patients were divided into four groups by DepOR (Q1 = 1-25\%, Q2 = 26-50\%, Q3 = 51-75\%, Q4 = 76-100\%). Kaplan-Meier curves were plotted for PFS against DepOR and the hazard ratio $(\mathrm{HR})$ was determined through univariable and multivariable cox regression models.

Results: In total, 265 patients were included for analysis. The number of patients in Group Q1-Q4 were 91 (34.3\%), 73 (27.5\%), 65 (24.5\%) and 36 (13.6\%), respectively. A greater DepOR was significantly associated with a longer PFS (Log-rank $P<0.0001)$. The HRs $(95 \% \mathrm{Cl}$ ) for PFS comparing patients with different DepOR status were $0.58(0.42-0.80)$ for Q2, $0.49(0.35-0.69)$ for Q3, and $0.33(0.22-0.50)$ for Q4, all compared with patients in Q1. DepOR as a continuous variable was also associated with prolonged PFS (HR, 0.20; $95 \% \mathrm{Cl}, 0.13-0.33$; $\mathrm{P}<0.001$ ). Additionally, in the multivariable cox regression model, abnormal $\mathrm{LDH}$, brain metastasis and male were found to be associated with worse PFS outcomes $(P<0.05)$.

Conclusion: A greater DepOR is significantly associated with PFS benefit in advanced NSCLC treated with EGFR-TKI, suggesting that it may be a useful clinical outcome to evaluate the response of targeted therapy.
\end{abstract}

Key words: DepOR, NSCLC, EGFR-TKI, targeted therapy

\section{Introduction}

Response Evaluation Criteria in Solid Tumors (RECIST) has been widely utilized to evaluate new therapeutic strategies in cancer. Objective response rate (ORR) could be used as an effective surrogate clinical endpoint for overall survival (OS) to predict tumors response via an anatomical approach with a unidimensional measurement of tumor burden according to the RECIST ${ }^{1}$. However, RECIST fails to assess the heterogeneity of response in highly active therapies, or to discern earlier read-out of activity for 
new therapeutics, and it also showed some limitations in predicting OS in some targeted therapies ${ }^{2-5}$.

In ALEX trial, no significant difference was observed between ORR in non-small cell lung cancer (NSCLC) patients treated with alectinib versus crizotinib ( $83 \%$ vs $76 \%$; $=0.09$ ). However, patients treated with alectinib obtained dramatically prolonged progression-free survival (PFS) compared with those treated with crizotinib ${ }^{3}$. Another trial with afatinib, an epidermal growth factor receptor (EGFR) tyrosine kinase inhibitor (TKI), showed a higher ORR (70\% vs $56 \%$; $=0.0083$ ) compared with gefitinib in NSCLC, however, such benefit was not observed for $\mathrm{OS}^{5}$. The above results suggested that alternative evaluation tools are needed to supplement RECIST with improved evaluation of clinical benefits in the NSCLC patients treated with targeted therapies.

The depth of response (DepOR), defined as the maximum percentage change in tumor size compared with baseline, has been indicated in several clinical trials to fetch up the insufficiency of RECIST in solid tumor evaluations. Increased DepOR were associated with a significantly longer post-progression survival (PPS) or OS in colorectal cancer patients treated with cetuximab or bevacizumab ${ }^{6-8}$. Choong-Kun Lee et al. further reported that DepOR could serve as a predictor for long-term outcome in advanced gastric cancer patients treated with trastuzumab ${ }^{9}$. The DepOR has also been investigated in the NSCLC patients treated with ALK inhibitor or anti-PD-1 antibody ${ }^{10}$. Altogether, the studies suggested DepOR to be an effective complement evaluation endpoint for PFS or OS.

Given the rapid development of targeted therapies in advanced NSCLC and the inadequacy of RECIST for monitoring treatment response, in this study, we aimed to provide a new strategy to assess disease response by evaluating the association between DepOR and PFS in NSCLC patients treated with EGFR-TKI.

\section{Materials and Methods}

\section{Patients}

A two-institution retrospective cohort analysis was performed at National Cancer Center/ Cancer Hospital of Chinese Academy of Medical Sciences and Cancer Center of Union Hospital, Tongji Medical College, Huazhong University of Science \& Technology. Advanced NSCLC patients harboring EGFR driver mutation (L858R or exon 19 deletion mutation) treated with EGFR-TKI (gefitinib, icotinib and erlotinib) as first-line treatment from August 2014 to July 2017 were included in this study. The exclusion criteria was: (1) Patients with malignant tumors history (except for basal cell or in situ cervical cancer and completely resected intramucosal gastric cancer) other than lung cancer; (2) Patients ever treated with EGFR TKI for postoperative adjuvant treatment or harboring EGFR T790M primary mutation; (3) Incomplete electronic medical records; (4) Patients without evaluable target lesion.

\section{Data collection}

For all patients, the following pretreatment demographic and clinical information were obtained from medical records: age, sex, TNM staging, pathology, EGFR mutation, smoking status, baseline lactic dehydrogenase (LDH), prior treatment, metastatic sites and combined therapy. Target lesion responses were assessed by two independent radiologists via computed tomography (CT) according to RECIST. DepOR was defined as maximum percent change in tumor size compared with baseline, patients were divided into four groups by maximal tumor shrinkage $(\mathrm{Q} 1=1-25 \%, \mathrm{Q} 2=$ $26-50 \%, \mathrm{Q} 3=51-75 \%, \mathrm{Q} 4=76-100 \%)$. PFS was defined as the interval from the start date of EGFR-TKI administration to the date of progression or death due to any cause.

\section{Statistical analysis}

Continuous variables were expressed as means and standard deviations (SDs) if normally distributed or median (range) if not normally distributed. Categorical variables were expressed as number and percentages. Survival probabilities were estimated using the Kaplan-Meier method, and a log-rank test was used to evaluate differences between survival curves. A Cox proportional hazards regression model was used for univariate and multivariate analyses. In the multivariable cox regression model including variables with $P$ value $<0.10$ in the univariate cox regression. All $\mathrm{P}$-values were two-sided, and $\mathrm{P}<0.05$ was considered statistically significant. Statistical analyses were performed with SPSS version 20 .

\section{Results}

\section{Patient demographics and characteristics}

The baseline demographics and characteristics for the patients are listed in Table 1. In total, 265 patients were included for the analyses, with 124 (46.8\%) from National Cancer Center/Cancer Hospital of Chinese Academy of Medical Sciences and 141 (53.2\%) from Cancer Center of Union Hospital, Tongji Medical College, Huazhong University of Science \& Technology. The median patient age was 61 (range 32-89) years, and $142(53.6 \%)$ patients were male. 258 patients $(97.4 \%)$ were adenocarcinoma, 256 $(96.7 \%)$ patients had an Eastern Cooperative 
Oncology Group (ECOG) performance status (PS) of $0-1$, and 157 (59.2\%) patients were never smokers. Patients were divided into four groups acoording to the maximal tumor shrinkage $(\mathrm{Q} 1=1-25 \%, \mathrm{Q} 2=$ $26-50 \%, \mathrm{Q} 3=51-75 \%, \mathrm{Q} 4=76-100 \%)$. The patient numbers for Group Q1-Q4 were 91 (34.3\%), 73 $(27.5 \%), 65(24.5 \%)$, and $36(13.6 \%)$, respectively. The patient demographics and disease characteristics were well-balanced across the quartiles for most variables except that patients in Group Q4 have a higher frequency of a normal lactate dehydrogenase level.

Table 1. Baseline demographics and characteristics of all patients.

\begin{tabular}{|c|c|c|c|c|c|}
\hline & $\begin{array}{l}\text { All }(n= \\
265)\end{array}$ & $\begin{array}{l}1-25 \%(\mathrm{n} \\
=91)\end{array}$ & $\begin{array}{l}26-50 \% \\
(n=73)\end{array}$ & $\begin{array}{l}51-75 \% \\
(\mathrm{n}=65)\end{array}$ & $\begin{array}{l}76-100 \% \\
(\mathrm{n}=36)\end{array}$ \\
\hline \multicolumn{6}{|l|}{ Sex, n (\%) } \\
\hline Male & $142(53.6)$ & $60(65.9)$ & $34(46.6)$ & $32(49.2)$ & $16(44.4)$ \\
\hline Female & $123(46.4)$ & $31(34.1)$ & $39(53.4)$ & $33(50.8)$ & $20(55.6)$ \\
\hline \multicolumn{6}{|l|}{ Age } \\
\hline Median & 61 & 61 & 60 & 59 & 63.5 \\
\hline Range & $32-89$ & $42-89$ & $32-78$ & $32-82$ & $38-82$ \\
\hline \multicolumn{6}{|l|}{ Stage } \\
\hline IIIB & $16(6)$ & $7(7.7)$ & $3(4.1)$ & $4(6.2)$ & $2(5.6)$ \\
\hline IV & $249(94)$ & $84(92.3)$ & $70(95.9)$ & $61(93.8)$ & $34(94.4)$ \\
\hline \multicolumn{6}{|l|}{ ECOG } \\
\hline 0 & $12(4.5)$ & $5(5.5)$ & $1(1.4)$ & $4(6.2)$ & $2(5.6)$ \\
\hline 1 & $244(92.1)$ & $83(91.2)$ & $70(95.9)$ & $59(90.8)$ & $32(88.9)$ \\
\hline 2 & $7(2.6)$ & $3(3.3)$ & $1(1.4)$ & $1(1.5)$ & $2(5.6)$ \\
\hline Unknown & $2(0.8)$ & $0(0)$ & $1(1.4)$ & $1(1.5)$ & $0(0)$ \\
\hline \multicolumn{6}{|l|}{ Smoking } \\
\hline Current & $43(16.2)$ & $18(19.8)$ & $12(16.4)$ & $9(13.8)$ & $4(11.1)$ \\
\hline Previous & $33(12.5)$ & $14(15.4)$ & $7(9.6)$ & $7(10.8)$ & $5(13.9)$ \\
\hline Never & $157(59.2)$ & $50(54.9)$ & $40(54.8)$ & $40(61.5)$ & $27(75)$ \\
\hline Unknown & $32(12.1)$ & $9(9.9)$ & $14(19.2)$ & $9(13.8)$ & $0(0)$ \\
\hline \multicolumn{6}{|l|}{ Pathology } \\
\hline adenocarcinoma & $258(97.4)$ & $91(100)$ & $70(95.9)$ & $62(95.4)$ & $36(100)$ \\
\hline squamous carcinoma & $3(1.1)$ & $0(0)$ & $2(2.7)$ & $1(1.5)$ & $0(0)$ \\
\hline adenosquamous carcinoma & $4(1.5)$ & $1(1.1)$ & $1(1.4)$ & $2(3.1)$ & $0(0)$ \\
\hline \multicolumn{6}{|l|}{ EGFR mutation } \\
\hline $19 \mathrm{del}$ & $134(50.6)$ & $39(42.9)$ & $32(43.8)$ & $41(63.1)$ & $22(61.1)$ \\
\hline L858R & $129(48.7)$ & $52(57.1)$ & $41(56.2)$ & $24(36.9)$ & $14(38.9)$ \\
\hline \multicolumn{6}{|l|}{ EGFR-TKI } \\
\hline geftinib & $106(40.0)$ & $44(48.4)$ & $21(28.8)$ & $26(40.0)$ & $15(41.7)$ \\
\hline icotinib & $125(47.2)$ & $34(37.4)$ & $43(58.9)$ & $31(47.7)$ & $17(47.2)$ \\
\hline erlotinib & $34(12.8)$ & $13(14.3)$ & $9(12.3)$ & $8(12.3)$ & $4(11.1)$ \\
\hline \multicolumn{6}{|l|}{ Metastasis } \\
\hline Liver & $52(19.6)$ & $18(19.8)$ & $17(23.3)$ & $11(16.9)$ & $6(16.7)$ \\
\hline Intrathoratic & $194(73.2)$ & $66(72.5)$ & $59(80.8)$ & $41(63.1)$ & $28(77.8)$ \\
\hline
\end{tabular}

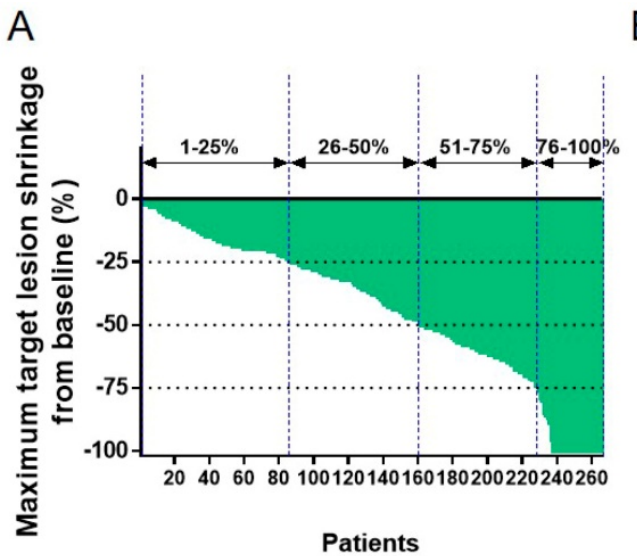

\begin{tabular}{llllll}
\hline & $\begin{array}{l}\text { All }(\mathrm{n}= \\
265)\end{array}$ & $\begin{array}{l}1-25 \%(\mathrm{n} \\
=91)\end{array}$ & $\begin{array}{l}26-50 \% \\
(\mathrm{n}=73)\end{array}$ & $\begin{array}{l}51-75 \% \\
(\mathrm{n}=65)\end{array}$ & $\begin{array}{l}76-100 \% \\
(\mathrm{n}=36)\end{array}$ \\
\hline Brain & $63(23.8)$ & $28(30.8)$ & $12(16.4)$ & $18(27.7)$ & $5(13.9)$ \\
Bone & $68(25.7)$ & $25(27.5)$ & $22(30.1)$ & $14(21.5)$ & $7(19.4)$ \\
Surgery & $67(25.3)$ & $20(22)$ & $14(19.2)$ & $16(24.6)$ & $17(47.2)$ \\
LDH & & & & & \\
$\quad$ Normal & $165(62.3)$ & $46(50.5)$ & $49(67.1)$ & $39(60.0)$ & $31(86.1)$ \\
Abnormal & $80(30.2)$ & $40(44.0)$ & $17(23.3)$ & $20(30.8)$ & $3(8.3)$ \\
Unknown & $20(7.5)$ & $5(5.5)$ & $7(9.6)$ & $6(9.2)$ & $2(5.6)$ \\
\hline
\end{tabular}

\section{Association between DepOR and PFS}

The association between DepOR and PFS was determined among patients in Groups Q1-Q4 that were divided upon the maximal tumor shrinkage as described above. The maximum tumor reduction for each patient was depicted in Figure 1A. The median PFS for patients from Groups Q1-Q4 were 6.8 months, 13.0 months, 13.7 months and 19.4 months, respectively $(\mathrm{P}<0.05)$. Kaplan-Meier survival curves were plotted for PFS against DepOR (Figure 1B), showing that a greater DepOR was significantly associated with a longer PFS (Log-rank $P<0.0001$ ).

\section{Univariate and multivariate analysis of progression-free survial of patients treated with EGFR-TKI}

In the univariate analysis, DepOR was significantly associated with PFS either as a rank variable (HR, 0.68; 95\% CI, 0.60-0.76; $\mathrm{P}<0.001$, Table 2) or as a continous variable (HR, $0.20 ; 95 \% \mathrm{CI}, 0.13-0.33$; $\mathrm{P}<0.001$, Table 2). In addition, sex, liver metastasis, brain metastasis, surgery prior to the use of TKI, and LDH level were associated or tended to be associated with the progression-free survival. Variables with a P value $<0.10$ in the univariate anlaysis were subsequently included in the multivariate cox regression model, showing that sex, brain metastasis, LDH level, and DepOR remained to be significantly associated with progression-free survival (Table 2).

B

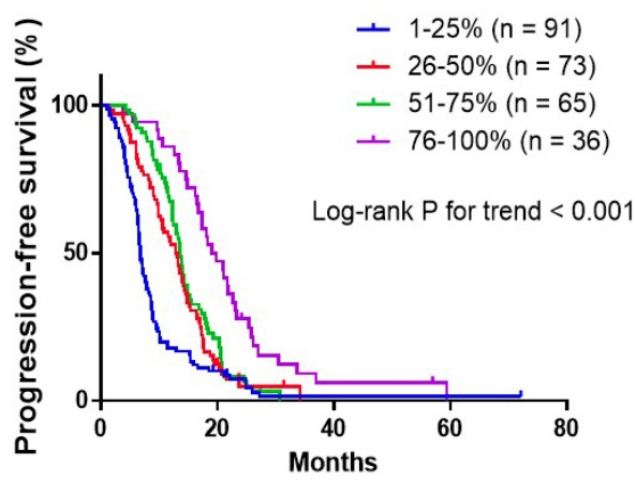

Figure 1. Association between depth of response and progression-free survival. (A) Waterfall plot depicting the maximum tumor shrinkage for each patient. (B) Kaplan-Meier survival curves of progression-free survival comparing patients with a maximum tumor shrinkage of $1-25 \%, 26-50 \%, 51-75 \%$ and $76-100 \%$. 
Table 2. Univariate and multivariate analyses of progression-free survival.

\begin{tabular}{|c|c|c|c|c|c|c|}
\hline & \multicolumn{3}{|c|}{ Univariate analysis } & \multicolumn{3}{|c|}{ Multivariate analysis } \\
\hline & HR & $95 \% \mathrm{CI}$ & $\mathrm{P}$ & HR & $95 \% \mathrm{CI}$ & $\mathrm{P}$ \\
\hline \multicolumn{7}{|l|}{ Sex } \\
\hline Male vs female & 1.27 & $0.99-1.62$ & 0.06 & 1.34 & $1.02-1.75$ & 0.04 \\
\hline \multicolumn{7}{|l|}{ Age } \\
\hline$\geq 65$ vs $<65$ & 1.1 & $0.85-1.43$ & 0.46 & & & \\
\hline \multicolumn{7}{|l|}{ Stage } \\
\hline IV vs IIIB & 0.92 & $0.54-1.55$ & 0.74 & & & \\
\hline \multicolumn{7}{|l|}{ EGFR driver mutation } \\
\hline L858R vs 19del & 1.07 & $0.83-1.37$ & 0.6 & & & \\
\hline \multicolumn{7}{|l|}{ Metastasis } \\
\hline Yes vs no & 1.04 & $0.60-1.83$ & 0.88 & & & \\
\hline \multicolumn{7}{|l|}{ Intrathoratic metastasis } \\
\hline Yes vs no & 0.83 & $0.63-1.10$ & 0.19 & & & \\
\hline \multicolumn{7}{|l|}{ Liver metastatic } \\
\hline Yes vs no & 1.41 & $1.03-1.93$ & 0.03 & 1.2 & $0.85-1.70$ & 0.29 \\
\hline \multicolumn{7}{|l|}{ Brain metastatic } \\
\hline Yes vs no & 1.83 & $1.37-2.46$ & $<0.001$ & 1.7 & $1.23-2.35$ & 0.001 \\
\hline \multicolumn{7}{|l|}{ bone_metastatic } \\
\hline Yes vs no & 1.22 & $0.92-1.62$ & 0.17 & & & \\
\hline \multicolumn{7}{|l|}{ Surgery } \\
\hline Yes vs no & 0.68 & $0.50-0.91$ & 0.008 & 0.98 & $0.71-1.34$ & 0.89 \\
\hline \multicolumn{7}{|l|}{ dNLR } \\
\hline$>3$ vs $\leq 3$ & 1.14 & $0.62-2.11$ & 0.67 & & & \\
\hline Lines of treatment & 0.96 & $0.74-1.24$ & 0.74 & & & \\
\hline DepOR (rank variable) & 0.68 & $0.60-0.76$ & $<0.001$ & 0.62 & $0.54-0.71$ & $<0.001$ \\
\hline DepOR (continuous variable) & 0.20 & $0.13-0.33$ & $<0.001$ & & & \\
\hline \multicolumn{7}{|c|}{$\mathrm{LDH}$} \\
\hline Abnoraml vs normal & 2.9 & $2.17-3.86$ & $<0.001$ & 2.82 & $2.08-3.82$ & $<0.001$ \\
\hline
\end{tabular}

Moreover, patients in Q1 were used as a comparative cohort for further analysis. Superior PFS were observed for patients in Groups Q2-Q4 when compared with patients in Q1 (Q2, HR $0.58,95 \% \mathrm{CI}$, 0.42-0.80, P<0.001; Q3, HR 0.49, 95\% CI, 0.35-0.69, $\mathrm{P}<0.001$; Q4: HR 0.33, 95\% CI, 0.22-0.50, P<0.001; Table 3). After adjusting the potential confounding factors including LDH level (normal vs abnormal), brain metastasis, and sex, DepOR was still an independent prognositc factor for PFS with a HR of $0.57 \quad(0.40-0.80, \quad \mathrm{P}<0.001)$ for $\mathrm{Q} 2,0.36 \quad(0.25-0.52$, $\mathrm{P}<0.001)$ for $\mathrm{Q} 3$ and $0.32(0.20-0.51, \mathrm{P}<0.001)$ for $\mathrm{Q} 4$ as compared with paitnets in Q1 (Table 3).

Table 3. Cox proportional hazard model for depth of response.

\begin{tabular}{llllllll}
\hline & \multicolumn{5}{c}{ Adjusted $^{*}$} \\
\hline & HR & $95 \%$ CI & P & HR & $95 \%$ CI & P \\
\hline $1-25 \%(\mathrm{n}=91)$ & & & & & & \\
$26-50 \%(\mathrm{n}=73)$ & 0.58 & $0.42-0.80$ & $<0.001$ & 0.57 & $0.40-0.80$ & 0.001 \\
$51-75 \%(\mathrm{n}=65)$ & 0.49 & $0.35-0.69$ & $<0.001$ & 0.36 & $0.25-0.52$ & $<0.001$ \\
$76-100 \%(\mathrm{n}=36)$ & 0.33 & $0.22-0.50$ & $<0.001$ & 0.32 & $0.20-0.51$ & $<0.001$ \\
\hline
\end{tabular}

*The HR was adjusted for sex, LDH, brain metastasis.

\section{Discussion}

In the present study, we have evaluated the relationship between DepOR and PFS in NSCLC patients treated with EGFR-TKI. The results demonstrated that a greater DepOR is associated with PFS benefit from EGFR-TKI treatment. Besides, brain metastasis, abnormal LDH level, and male were associated with worse PFS.

OS is currently the golden standard for oncology clinical trial endpoints ${ }^{11}$, however, it is inefficient and time-consuming for clinical evaluation, thus finding a surrogated early endpoint is important for clinical assessment. ORR evaluated by RECIST criteria has been widely applied by clinicians. However, previous studies revealed that ORR may fail to capture the full benefit of treatment ${ }^{11}$ and discern earlier read-out of activity for new therapeutics. In addition, ORR outcome is not always consistent with PFS or OS in evaluating endpoints in oncology $y^{3,4}$. Under these circumstances, DepOR has been proposed as a complement factor for ORR to evaluate the clinical benefit ${ }^{7}$.

Investigations have been carried out in several solid tumors using DepOR as a clinical endpoint. In the phase III trial comparing cetuximab in combination with FOLFIRI as first-line treatments in colorectal cancer patients, an increased DepOR, while not ORR, was associated with a longer OS in patients treated with cetuximab plus FOLFIRI ${ }^{6}$. DepOR has also been studied in a post-hoc analysis of CRYSTAL and OPUS trials in colon cancer, in which a greater DepOR was demonstrated to be associated with longer OS and post-progression survival ${ }^{8}$. Another clinical trial showed that gastric cancer patients with a DepOR $\geq 45 \%$ obtained longer PFS and OS when treated with trastuzumab ${ }^{9}$. In fact, the satisfactory performance of DepOR as an evaluation factor has not only been shown for targeted therapies, but also for immunotherapy in NSCLC ${ }^{10}$. In addition, DepOR has been investigated in hematologic malignancies to evaluate the changes of M-protein in multiple myeloma and minimal residual disease in leukemia and myeloma ${ }^{12-15}$, implying the validity of DepOR as a complementary endpoint established for different interventions and different neoplasms.

In the present study, we demonstrated that a greater DepOR was significantly associated with a longer PFS. After adjustment of potential confounding factors including LDH level, brain metastasis, and sex, DepOR was still an independent prognositc factor for PFS. We have proved that DepOR could serve as an effective complementary clinical endpoint to evaluate the clinical benefit of EGFR TKI in patients with NSCLC.

Besides, we showed that an abnormal lactate dehydrogenase level was associated with worse PFS. LDH has been widely used as a classic inflammatory marker for lung cancer patients treated with chemotherapy or targeted therapies ${ }^{16-18}$. One previous study demonstrated that an abnormal LDH level might be a negative predictor of survival in NSCLC 
patients receiving immune checkpoint blockades ${ }^{19}$. However, our study revealed that the abnormal LDH was associated with shorter PFS in advanced NSCLC patients treated with EGFR-TKI, suggesting that LDH may be an independent prognosis indicator for NSCLC instead of a predictive biomarker for immunotherapy.

We also found that brain metastasis was a potential poor prognostic factor independent of the DepOR. Indeed, EGFR-TKIs administrated in this study, including gefitinib, icotinib and erlotinib, have been evaluated in patients with brain metastasis with a mild benefit ${ }^{20-22}$, probably because of their poor capability to penetrate the blood-brain barrier. For a majority of the NSCLC patients with central nervous system (CNS) metastasis, brain metastasis was the first site of treatment failure after initial response ${ }^{23}$, supporting the aforementioned blood-brain barrier hypothesis.

Our study has provided a new strategy to evaluate the clinical outcomes, however, there are still some limitations need to be considered. Firstly, this is a retrospective study that may yield selecting bias. Nevertheless, the patient demographics and disease characteristics were well-balanced across the quartiles for most variables. Secondly, the association between DepOR and OS was not analyzed due to the consideration of the changes of treatment for patients upon disease progression, which may introduce potential bias to the results. Thirdly, in our study, the association between DepOR and PFS was conducted only in patients with tumor shrinkage due to the limited number of patients with progressed disease upon EGFR-TKI. Last but not least, the ability of response assessment between DepOR and RECIST was not compared directly, a larger prospective study is warranted for further studies. In summary, we have demonstrated that DepOR was associated with better PFS either as a rank variable or a continuous variable, suggesting that the DepOR may be a supplement to RECIST to evaluate the treatment response in future clinical practice.

\section{Conclusion}

The DepOR may be a useful clinical outcome to efficiently evaluate the response of EGFR-TKI in patients with NSCLC. More evidences are needed to understand how to use DepOR as an endpoint in clinical trials.

\section{Abbreviations}

CT: computed tomography; DepOR: depth of response; EGFR: epidermal growth factor receptor; ECOG: Eastern Cooperative Oncology Group; LDH: lactic dehydrogenase; NSCLC: no-small cell lung cancer; ORR: objective response rate; OS: overall survival; PS: performance status; PFS: progress free survival; PPS: post-progression survival; RECIST: Response Evaluation Criteria in Solid Tumors; SDs: standard deviations; TKI: tyrosine kinase inhibitors.

\section{Acknowledgments}

This work was supported by National Key Technology Support Program (2014BAI09B01) and Chinese National Major Project for New Drug Innovation (2017ZX09304015).

\section{Competing Interests}

The authors have declared that no competing interest exists.

\section{References}

1. Wilson MK, Karakasis K, Oza AM. Outcomes and endpoints in trials of cancer treatment: the past, present, and future. The Lancet. Oncology. Jan 2015;16(1):e32-42.

2. Hodi FS, Hwu WJ, Kefford R, et al. Evaluation of Immune-Related Response Criteria and RECIST v1.1 in Patients With Advanced Melanoma Treated With Pembrolizumab. Journal of clinical oncology : official journal of the American Society of Clinical Oncology. May 1 2016;34(13):1510-1517.

3. Gadgeel S, Peters S, Mok T, et al. Alectinib versus crizotinib in treatment-naive anaplastic lymphoma kinase-positive (ALK+) non-small-cell lung cancer: CNS efficacy results from the ALEX study. Annals of oncology : official journal of the European Society for Medical Oncology. Nov 1 2018;29(11):2214-2222.

4. Aix SP, Park K, Tan EH, et al. P1.34: First-Line Afatinib vs Gefitinib for Patients With EGFR Mutation-Positive Non-Small-Cell Lung Cancer: The LUX-Lung 7 Trial: Track: Advanced NSCLC. Journal of thoracic oncology : official publication of the International Association for the Study of Lung Cancer. Oct 2016;11(10s):S203-s204.

5. Park K, Tan EH, O'Byrne K, et al. Afatinib versus gefitinib as first-line treatment of patients with EGFR mutation-positive non-small-cell lung cancer (LUX-Lung 7): a phase 2B, open-label, randomised controlled trial. The Lancet, Oncology. May 2016;17(5):577-589.

6. Stintzing S, Modest DP, Rossius L, et al. FOLFIRI plus cetuximab versus FOLFIRI plus bevacizumab for metastatic colorectal cancer (FIRE-3): a post-hoc analysis of tumour dynamics in the final RAS wild-type subgroup of this randomised open-label phase 3 trial. The Lancet. Oncology. Oct 2016;17(10):1426-1434.

7. Cremolini C, Loupakis F, Antoniotti C, et al. Early tumor shrinkage and depth of response predict long-term outcome in metastatic colorectal cancer patients treated with first-line chemotherapy plus bevacizumab: results from phase III TRIBE trial by the Gruppo Oncologico del Nord Ovest. Annals of oncology : official journal of the European Society for Medical Oncology. Jun 2015;26(6):1188-1194

8. Mansmann UR, Sartorius U, Laubender RP, Giessen CA, Esser R, Heinemann V. Quantitative analysis of the impact of deepness of response on post-progression survival time following first-line treatment in patients with mCRC. Journal of Clinical Oncology. 2013;31(15_suppl):3630-3630.

9. Lee CK, Kim SS, Park S, et al. Depth of response is a significant predictor for long-term outcome in advanced gastric cancer patients treated with trastuzumab. Oncotarget. May 9 2017;8(19):31169-31179.

10. McCoach CE, Blumenthal GM, Zhang L, et al. Exploratory analysis of the association of depth of response and survival in patients with metastatic non-small-cell lung cancer treated with a targeted therapy or immunotherapy. Ann Oncol, 2019. 30(3): p. 492

11. Eisenhauer EA, Therasse P, Bogaerts J, et al. New response evaluation criteria in solid tumours: revised RECIST guideline (version 1.1). European journal of cancer. Jan 2009; 45(2):228-247.

12. Kumar S, Paiva B, Anderson KC, et al. International Myeloma Working Group consensus criteria for response and minimal residual disease assessment in multiple myeloma. The Lancet. Oncology. Aug 2016;17(8):e328-e346.

13. Herold M, Haas A, Srock S, et al. Rituximab added to first-line mitoxantrone, chlorambucil, and prednisolone chemotherapy followed by interferon maintenance prolongs survival in patients with advanced follicular lymphoma: an East German Study Group Hematology and Oncology Study. Journal of clinical oncology: official journal of the American Society of Clinical Oncology. May 20 2007;25(15):1986-1992.

14. Walter RB, Gooley TA, Wood BL, et al. Impact of pretransplantation minimal residual disease, as detected by multiparametric flow cytometry, on outcome of myeloablative hematopoietic cell transplantation for acute myeloid 
leukemia. Journal of clinical oncology : official journal of the American Society of Clinical Oncology. Mar 20 2011;29(9):1190-1197.

15. Lonial S, Anderson KC. Association of response endpoints with survival outcomes in multiple myeloma. Leukemia. Feb 2014;28(2):258-268.

16. Petrelli F, Cabiddu M, Coinu A, et al. Prognostic role of lactate dehydrogenase in solid tumors: a systematic review and meta-analysis of 76 studies. Acta oncologica (Stockholm, Sweden). Jul 2015;54(7):961-970.

17. Fiala O, Pesek M, Finek J, et al. Change in Serum Lactate Dehydrogenase Is Associated with Outcome of Patients with Advanced-stage NSCLC Treated with Erlotinib. Anticancer research. May 2016;36(5):2459-2465.

18. Inomata $\mathrm{M}$, Hayashi $\mathrm{R}$, Tanaka $\mathrm{H}$, et al. Elevated levels of plasma lactate dehydrogenase is an unfavorable prognostic factor in patients with epidermal growth factor receptor mutation-positive non-small cell lung cancer, receiving treatment with gefitinib or erlotinib. Molecular and clinical oncology. May 2016;4(5):774-778.

19. Mezquita L, Auclin E, Ferrara R, et al. Association of the lung immune prognostic index with immune checkpoint inhibitor outcomes in patients with advanced non-small cell lung cancer. JAMA Oncology. 2018;4(3):351-357.

20. Kelly WJ, Shah NJ, Subramaniam DS. Management of Brain Metastases in Epidermal Growth Factor Receptor Mutant Non-Small-Cell Lung Cancer. Frontiers in oncology. 2018;8:208.

21. Bai H, Xiong L, Han B. The effectiveness of EGFR-TKIs against brain metastases in EGFR mutation-positive non-small-cell lung cancer. OncoTargets and therapy. 2017;10:2335-2340.

22. Biaoxue R, Hua L, Wenlong G, Shuanying Y. Efficacy and safety of icotinib in treating non-small cell lung cancer: a systematic evaluation and meta-analysis based on 15 studies. Oncotarget. 2016;7(52):86902-86913.

23. Economopoulou P, Mountzios G. Non-small cell lung cancer (NSCLC) and central nervous system (CNS) metastases: role of tyrosine kinase inhibitors (TKIs) and evidence in favor or against their use with concurrent cranial radiotherapy. Translational lung cancer research. 2016;5(6):588-598. 